Bulletin of the Seismological Society of America. Vol. 65, No. 6, pp. 1753-1760. December 1975

\title{
A PERTURBATION SCHEME FOR OBTAINING PARTIAL DERIVATIVES OF LOVE-WAVE GROUP-VELOCITY DISPERSION
}

\author{
By DAN KOSLOFF
}

\section{ABSTRACT}

A method is derived for obtaining partial derivatives of Love-wave groupvelocity spectra for a layered medium using a second-order perturbation theory. These partials are a prerequisite for systematic inversion of group-velocity spectra but they are helpful as well in trial and error methods. Mathematically the equation of motion and boundary conditions for Love waves are a singular Sturm Liouville type eigenvalue problem. In the case of a fixed wave number, the eigenvalues are the negative of the square of the frequencies. Thus, by expressing the first- and second-order perturbations of the eigenvalues in terms of partial derivatives of the frequency with respect to the wave number and material parameters of the medium, one can relate these perturbations to groupvelocity partials. The scheme should be relatively economical and easy to incorporate in Love-wave dispersion codes.

\section{INTRODUCTION}

Group- and phase-velocity dispersion of Love waves have proved to be a strong tool in earth-structure determination. When attempting to perform these studies by systematic inversion of the data, one has to be able to establish the relations between small perturbations in the material parameters of the medium, and the resulting changes in the dispersion curves. These formulas are also useful in trial and error methods as they give an indication in which direction the model needs to be changed in order to produce a closer fit between observations and the theoretical results.

By means of variational methods or the equivalent first-order perturbation theory, these relations have been derived for Love-wave phase velocity and expressed in terms of partial derivatives of the phase velocity with respect to the density and shear modulus of each of the layers used to approximate the medium (Takeuchi et al., 1964; Anderson, 1964; Anderson and Harkrider, 1968; Harkrider, 1968). For group velocity, the method to obtain the partials has been to take differences between dispersion curves corresponding to structures differing in one parameter in a certain layer, or to use the formulation of Anderson ard Harkrider (1968). In the Anderson and Harkrider formulation, the partials are derived from partials of the energy integrals, arrived at by calculating derivatives of the entries in the Thomson-Haskell matrix. Instead of the two previous methods, this paper presents a second-order perturbation scheme to obtain the Lovewave group-velocity partials. This method should be more economical, especially for a large number of layers.

There are a number of reasons why under certain circumstances it may be desirable to use group-velocity dispersion for structure studies instead of or in addition to phasevelocity dispersion. Often, the data that are first produced from the observations are group-velocity dispersion, and hence the ability to invert those data directly avoids the necessity of integrating and converting them to phase-velocity dispersion. Second, in most cases, group-velocity curves have a less regular form than the corresponding phasevelocity curves, in terms of slope, changes, and inflection points. Moreover, as will be 
demonstrated later, group-velocity partials often change sign; for certain wave numbers and depths an increase in shear velocity in a particular layer can result in a decrease in the group velocity (Anderson and Harkrider, 1968). These properties make the task of fitting group-velocity dispersion more difficult, but when the fit is achieved there is a large probability that the model resembles the actual medium. Moreover, when fitting group velocity and phase velocity simultaneously, one achieves, in a sense, a fitting of a function and its first derivative, which is an improvement over fitting the function only.

\section{THEORY}

The equation of motion and boundary conditions for Love waves in a medium in which the density and shear modulus vary only in the vertical $Z$ direction are given by

$$
\begin{gathered}
\frac{d}{d z}\left(\mu \frac{d U_{l}}{d z}\right)-k^{2} \mu U_{l}=-\rho \omega_{l}^{2} U_{l} ; \\
\left.\mu \frac{d U_{l}}{d z}\right|_{z=0}=\left.0 \quad U_{l}\right|_{z=\infty}=0
\end{gathered}
$$

where $U_{l}$ is the horizontal transverse displacement of the $l$ th mode, $\mu$ the shear modulus, $\rho$ the density, $k$ the wave number and $\omega_{l}$ the frequency of the $l$ th mode. It is assumed that $\rho$ and $\mu$ are piecewise continuous functions of $z$.

Regarding $\mu, \rho$ and $k$ as parameters, the set of equations (1) and (2) becomes a singular self-adjoint boundary value problem on the interval $(0, \infty)$ with eigenvalues $\lambda_{l}=-\omega_{l}^{2}$ (Coddington and Levinson, Ch. 9) and eigenfunctions $U_{l}$. The solutions of (1) and (2) consist of a finite set of eigenfunctions $U_{l}$ with discrete and distinct eigenvalues which fulfill the inequality

$$
\left|\lambda_{1}\right|<\left|\lambda_{2}\right|<\ldots\left|\lambda_{N}\right| \ldots
$$

and beyond a critical value $|\lambda|>\lambda_{c}$ corresponding to the shear velocity of the halfspace eigenfunctions with a continuous distribution of eigenvalues. The former are known as "locked" modes and the latter correspond to "leaky modes".

The derivation of group-velocity partials follows closely standard perturbation theory. We consider a small change in the parameters of the problem $\rho \rightarrow \rho+\delta \rho, \mu \rightarrow \mu+\delta \mu$, $k \rightarrow k+\delta k$ as a result of which the eigenfunctions $\left\{U_{l}\right\}_{l=1}^{\infty}$ and eigenvalues $\left\{\lambda_{l}\right\}_{l=1}^{\infty}$ will assume slightly different values. The new eigenfunction and eigenvalues can be expanded in a series

$$
\begin{gathered}
U_{l}(z)=U_{l}^{(0)}(z)+U_{l}^{(1)}(z)+U_{l}^{(2)}(z)+\ldots \\
\lambda_{l}=\lambda_{l}{ }^{(0)}+\lambda_{l}{ }^{(1)}+\lambda_{l}^{(2)}+\ldots
\end{gathered}
$$

where the terms with zero superscripts correspond to the original unperturbed model and the terms with an $n$th superscript are of the order of the $n$th power of the perturbations. All the $U_{i}{ }^{(i)}$ functions in (4) fulfill the boundary conditions (2).

We define an inner product of two functions $U$ and $V$ by

$$
[u, v]=\int_{0}^{\infty} u v d z
$$

Using this definition, the orthogonality of the displacement functions of the various Love modes can be expressed as

$$
\left[\rho U_{m}, U_{i}\right] /\left[\rho U_{l}, U_{l}\right]=\delta_{m l} .
$$

Another property of the eigenfunctions of Love waves and of solutions of self-adjoint 
boundary value problems, in general, is that the eigenfunctions form a complete set so that any function $V(z)$ satisfying the boundary conditions (2) can be expanded by

$$
V(z)=\sum_{m=0}^{\infty} C_{m} U_{m}(z)
$$

The sum notation represents an integration for the continuous part of the spectrum and an ordinary sum over the discrete part. The expansion coefficients in $C_{m}$ in (8) are given by

$$
C_{m}=\left[\rho V(z), U_{m}(z)\right] /\left[\rho U_{m}(z), U_{m}(z)\right] .
$$

Using this properly, all the perturbed functions $U_{l}^{(1)}(z)$ can be expanded by

$$
U_{l}^{(i)}(z)=\sum_{m=0}^{\infty} C_{m}{ }^{(i)} U_{m}{ }^{(0)}(z)
$$

with

$$
C_{m}{ }^{(i)}=\left[\rho U_{l}^{(i)}(z), U_{m}{ }^{(0)}(z)\right] /\left[\rho U_{m}^{(0)}, U_{m}{ }^{(0)}\right] .
$$

Rewriting equation (1) for the perturbed state results in,

$$
\begin{aligned}
& \frac{d}{d z}\left[(\mu+\delta \mu) \frac{d}{d z}\left(U_{l}^{(0)}+U_{l}^{(1)}+\ldots\right)\right]-(k+\delta k)^{2}(\mu+\delta \mu)\left(U_{l}^{(0)}+U_{l}^{(1)}+\ldots\right) \\
& \quad=-(\rho+\delta \rho)\left(\lambda_{l}{ }^{(0)}+\lambda_{l}{ }^{(1)}+\ldots\right)\left(U_{l}^{(0)}+U_{l}^{(1)}+\ldots\right) .
\end{aligned}
$$

After multiplying this equation from the left by $U_{m}{ }^{(0)}$ and forming the inner product, then integrating by parts and rearranging terms using the boundary conditions (2), one gets

$$
\begin{aligned}
& -\left[(\mu+\delta \mu) \frac{d U_{m}^{(0)}}{d z}, \frac{d}{d z}\left(U_{l}^{(0)}+U_{l}^{(1)}+\ldots\right)\right] \\
& -(k+\delta k)^{2}\left[(\mu+\delta \mu) U_{m}{ }^{(0)},\left(U_{l}^{(0)}+U_{l}^{(1)}+\ldots\right)\right] \\
& =\left(\lambda_{l}^{(0)}+\lambda_{l}^{(1)}+\ldots\right)\left[(\rho+\delta \rho) U_{m}^{(0)},\left(U_{l}^{(0)}+U_{l}^{(1)}+\ldots\right)\right] .
\end{aligned}
$$

In equation (13) the sum of the terms of the same order in the perturbation should be equal on both sides, therefore equating terms of the first order with $m=l$ results in

$$
\begin{aligned}
-\left[\mu \frac{d U_{l}^{(0)}}{d z}, \frac{d U_{l}^{(1)}}{d z}\right]- & k^{2}\left[\mu U_{l}^{(0)}, U_{l}^{(1)}\right]-\left[\delta \mu \frac{d U_{l}^{(0)}}{d z}, \frac{d U_{l}^{(0)}}{d z}\right]-2 k \delta k\left[\mu U_{l}^{(0)}, U_{l}^{(0)}\right] \\
-k^{2}\left[\delta \mu U_{l}^{(0)}, U_{l}^{(0)}\right]= & \lambda_{l}^{(0)}\left[\rho U_{l}^{(0)}, U_{l}^{(1)}\right]+\lambda_{l}^{(0)}\left[\delta \rho U_{l}^{(0)}, U_{l}^{(0)}\right] \\
& +\lambda_{l}^{(1)}\left[\rho U_{l}^{(0)}, U_{l}^{(0)}\right] .
\end{aligned}
$$

All the terms in (14) containing $U_{l}^{(1)}$ and its derivatives cancel on both sides after integrating by parts and using the equation of motion (1). By rearranging terms we obtain the expression for the first perturbation in $\lambda_{l}$

$$
\lambda_{l}^{(1)}=\frac{-\left[\delta \mu \frac{d U_{l}^{(0)}}{d z}, \frac{d U_{I}^{(0)}}{d z}\right]-k^{2}\left[\delta \mu U_{l}^{(0)} U_{l}^{(0)}\right]-2 k \delta k\left[\mu U_{l}^{(0)}, U_{l}^{(0)}\right]}{-\lambda_{l}^{(0)}\left[\delta \rho U_{l}^{(0)}, U_{l}^{(0)}\right]} .
$$

This result has been obtained previously by a number of authors (Anderson and Archambeau, 1964; Anderson, 1964) by variational methods. 
Equating first-order terms in (13) with $m \neq l$ gives after a similar procedure of rearranging the terms

$$
\left[\rho U_{m}{ }^{(0)}, U_{l}^{(1)}\right]=\frac{-\left[\delta \mu \frac{d U_{m}{ }^{(0)}}{d z}, \frac{d U_{l}^{(0)}}{d z}\right]-2 k \delta k\left[\mu U_{m}{ }^{(0)}, U_{l}^{(0)}\right]-k^{2}\left[\delta \mu U_{m}{ }^{(0)}, U_{l}^{(0)}\right]}{-\lambda_{l}^{(0)}\left[\delta \rho U_{m}{ }^{(0)}, U_{l}^{(0)}\right]} .
$$

Combining (11) and (16) will give the expressions for $C_{m}{ }^{(1)}$ with $m \neq l$. The coefficient $C_{l}{ }^{(1)}$ is as yet undetermined but can be set to zero without causing any first-order errors (Landau and Lifshitz). After equating second-order terms in equation (13) for $m=l$ all the terms with $U_{l}^{(2)}$ and its derivatives will cancel on both sides in a similar manner as in deriving (14). Finally, after a number of algebraic steps, the formula for the second-order perturbation in $\lambda_{l}$ is obtained

$$
\lambda_{l}^{(2)}=\frac{-\left[\delta \mu \frac{d U_{l}^{(0)}}{d z}, \frac{d U_{l}^{(1)}}{d z}\right] \begin{array}{c}
-k^{2}\left[\delta \mu U_{l}^{(0)}, U_{l}^{(1)}\right]-2 k \delta k\left[\mu U_{l}^{(0)}, U_{l}^{(1)}\right] \\
-2 k \delta k\left[\delta \mu U_{l}^{(0)}, U_{l}^{(0)}\right]-\delta k^{2}\left[\mu U_{l}^{(0)}, U_{l}^{(0)}\right] \\
-\lambda_{l}^{(0)}\left[\delta \rho U_{l}^{(0)}, U_{l}^{(0)}\right]-\lambda_{l}^{(1)}\left[\delta \rho U_{l}^{(0)}, U_{l}^{(0)}\right]
\end{array}}{\left[\rho U_{l}^{(0)}, U_{l}^{(0)}\right]}
$$

Substituting from (15) (11) and (16) into (17), gives

$$
\begin{aligned}
\lambda_{l}^{(2)}= & \frac{1}{\left[\rho U_{l}^{(0)}, U_{l}^{(0)}\right]} \\
& \times\left\{\sum_{\substack{m=1 \\
m \neq l}}^{\infty} \frac{4 k \delta k\left[\mu U_{l}^{(0)}, U_{m}{ }^{(0)}\right]\left\{\left[\delta \mu \frac{\left.\left.d U_{l}^{(0)}, \frac{d U_{m}{ }^{(0)}}{d z}\right]+k^{2}\left[\delta \mu U_{l}^{(0)}, U_{m}{ }^{(0)}\right]\right\}}{\left(\lambda_{l}{ }^{(0)}-\lambda_{m}{ }^{(0)}\right)\left[\rho U_{m}{ }^{(0)}, U_{m}^{(0)}\right]}\right.\right.}{}\right. \\
& +\sum_{\substack{m=1 \\
m \neq l}}^{\infty} \frac{4 k \delta k\left[\mu U_{l}^{(0)}, U_{m}{ }^{(0)}\right] \lambda_{l}^{(0)}\left[\delta \rho U_{m}{ }^{(0)}, U_{l}^{(0)}\right]}{\left(\lambda_{l}^{(0)}-\lambda_{m}{ }^{(0)}\right)\left[\rho U_{m}{ }^{(0)}, U_{m}{ }^{(0)}\right]}+\frac{2 k \delta k\left[\mu U_{l}^{(0)}, U_{l}^{(0)}\right]\left[\delta \rho U_{l}^{(0)}, U_{l}^{(0)}\right]}{\left[\rho U_{l}^{(0)}, U_{l}^{(0)}\right]} \\
& \left.-2 k \delta k\left[\delta \mu U_{l}^{(0)}, U_{l}^{(0)}\right]\right\}+R .
\end{aligned}
$$

In $R$, for the sake of brevity, we included all the second-order terms not containing products of $\delta \rho \delta k$ or $\delta \mu \delta k$ (terms like $\delta k^{2}$ etc.) which will not be used later. It should be noted that in addtion to the second-order terms encountered in standard perturbation theory which involve an inner product between different modes, the formula (18) contains terms which do not couple different modes. This arises from the fact that the perturbation operator itself has second-order terms in it.

Equations (15) and (18) are applicable to any medium with piecewise continuous material parameters. In the case where the medium is approximated by a number of homogeneous layers over a half-space, the results can be more conveniently expressed in terms of partial derivatives of the eigenvalues $\lambda_{l}$ with respect to the various parameters of the problem. For the case of $\mathrm{N}-1$ layers over a half-space all the inner products can be written as

$$
[U, V]=\sum_{s=1}^{N}[U, V]^{s}
$$


where

$$
[U, V]^{s}=\int_{z_{s}}^{z_{s+1}} U V d z
$$

where $z_{s}$ is the vertical distance from the free surface to the top of the sth layer and $z_{N+1}=\infty$.

In the case of $N-1$ layers over a half-space, each $\delta \mu_{s} \delta \rho_{s}$ is constant within the $s$ th layer. Hence we can express the perturbed eigenvalues $\lambda_{l}$ in (5) as a Taylor series

$$
\begin{aligned}
\lambda_{l}= & \lambda_{l}{ }^{(0)}+\frac{\partial \lambda_{l}}{\partial k} \delta k+\sum_{s=1}^{N} \frac{\partial \lambda_{l}}{\partial \rho_{s}} \delta \rho_{s}+\sum_{s=1}^{N} \frac{\partial \lambda_{l}}{\partial \mu_{s}} \delta \mu_{s}+\sum_{s=1}^{N} \frac{\partial^{2} \lambda_{l}}{\partial \mu_{s} \partial k} \delta \mu_{s} \delta k \\
& +\sum_{s=1}^{N} \frac{\partial^{2} \lambda_{l}}{\partial \rho_{s} \partial k} \delta k \delta \rho_{s}+\frac{1}{2} \sum_{\substack{s=1 \\
q=1}}^{N} \frac{\partial^{2} \lambda_{l}}{\partial \rho_{s} \partial \mu_{q}} \delta \rho_{s} \delta \mu_{q}+\ldots
\end{aligned}
$$

It is understood that when taking a derivative with respect to one variable all the other variables are held constant.

Comparing (5), (15), (18), (21) yields

$$
\begin{aligned}
& \frac{\partial \lambda_{l}}{\partial k}=\frac{-2 k\left[\mu U_{l}^{(0)}, U_{l}^{(0)}\right]}{\left[\rho U_{l}^{(0)}, U_{l}^{(0)}\right]}, \\
& \frac{\partial \lambda_{l}}{\partial \rho_{s}}=\frac{-\lambda_{l}{ }^{(0)}\left[U_{l}^{(0)}, U_{l}^{(0)}\right]^{s}}{\left[\rho U_{l}^{(0)}, U_{l}^{(0)}\right]} \\
& \frac{\partial \lambda_{l}}{\partial \mu_{s}}=-\frac{\left[\frac{d U_{l}^{(0)}}{d z}, \frac{d U_{l}^{(0)}}{d z}\right]^{s}-k^{2}\left[U_{l}^{(0)}, U_{l}^{(0)}\right]^{s}}{\left[\rho U_{l}^{(0)}, U_{l}^{(0)}\right]} \\
& \frac{\partial^{2} \lambda_{l}}{\partial k \partial \mu_{s}}=\frac{-2 k\left[U_{l}^{(0)}, U_{l}^{(0)}\right]^{s}}{\left[\rho U_{l}^{(0)}, U_{l}^{(0)}\right]}+\frac{1}{\left[\rho U_{l}^{(0)}, U_{l}^{(0)}\right]} \\
& \times \sum_{\substack{m=1 \\
m \neq l}}^{\infty} \frac{4 k\left[\mu U_{l}^{(0)}, U_{m}{ }^{(0)}\right]\left\{\left[\frac{d U_{l}^{(0)}}{d z}, \frac{d U_{l}^{(0)}}{d z}\right]^{s}+k^{2}\left[U_{l}^{(0)}, U_{l}{ }^{(0)}\right]^{s}\right\}}{\left(\lambda_{l}{ }^{(0)}-\lambda_{m}{ }^{(0)}\right)\left[\rho U_{m}{ }^{(0)}, U_{m}{ }^{(0)}\right]} \\
& \frac{\partial^{2} \lambda_{l}}{\partial k \partial \rho_{s}}=\frac{2 k\left[\mu U_{l}^{(0)}, U_{l}^{(0)}\right]\left[U_{l}^{(0)}, U_{l}^{(0)}\right]^{s}}{\left[\rho U_{l}^{(0)}, U_{l}^{(0)}\right]\left[\rho U_{l}^{(0)}, U_{l}^{(0)}\right]}+\frac{1}{\left[\rho U_{l}^{(0)}, U_{l}^{(0)}\right]} \\
& \times \sum_{\substack{m=1 \\
m \neq l}}^{\infty} \frac{4 k \lambda_{l}{ }^{(0)}\left[\mu U_{l}{ }^{(0)}, U_{m}{ }^{(0)}\right]\left[U_{m}{ }^{(0)}, U_{l}^{(0)}\right]^{s}}{\left(\lambda_{l}{ }^{(0)}-\lambda_{m}{ }^{(0)}\right)\left[\rho U_{m}{ }^{(0)}, U_{m}{ }^{(0)}\right]}
\end{aligned}
$$

In order to obtain the partials of the group velocity from (22) to (26) we recall that by definition $V_{g}=\partial \omega / \partial k$, and therefore,

$$
\begin{aligned}
V_{g l} & =-\frac{1}{2 \omega_{l}} \frac{\partial \lambda_{l}}{\partial k} \\
\frac{\partial V_{g l}}{\partial \rho_{s}} & =-\frac{1}{2 \omega_{l}}\left(\frac{\partial^{2} \lambda_{l}}{\partial k \partial \mu_{s}}+\frac{1}{2 \omega_{l}^{2}} \frac{\partial \lambda_{l}}{\partial \mu_{s}} \cdot \frac{\partial \lambda_{l}}{\partial k}\right) \\
\frac{\partial V_{g l}}{\partial \mu_{s}} & =-\frac{1}{2 \omega_{l}}\left(\frac{\partial^{2} \lambda_{l}}{\partial k \partial \rho_{s}}+\frac{1}{2 \omega_{l}^{2}} \frac{\partial \lambda}{\partial \rho_{s}} \frac{\partial \lambda_{l}}{\partial k}\right) .
\end{aligned}
$$

Combining (22) to (26) with (27) to (29) yields the desired partial derivatives of the Love-wave group velocity. 


\section{ANALytical AND Numerical Results}

For a single layer of thickness $d$, density $\rho$ and shear modulus $\mu$ overlying an infinitely rigid half-space, the solution of equations (1) and (2) has a simple closed form

$$
U_{l}(z)=\operatorname{Cos}\left(\left(l-\frac{1}{2}\right) \frac{\pi z}{d}\right), \quad l=1,2 \ldots
$$

The eigenvalues are given by

$$
\lambda_{l}=-\frac{\mu}{\rho} k^{2}+\left(\left(l-\frac{1}{2}\right) \frac{\pi}{d}\right)^{2} .
$$

As a result of the orthogonality of the cosine functions in (30) over the interval $(0, d)$ and of their derivatives (which are sine functions), all the terms with $m \neq l$ in (18) will cancel. Substituting (30) into (22) to (26) yields

$$
\begin{gathered}
\frac{\partial \lambda_{l}}{\partial k}=-\frac{2 k \mu}{\rho} \\
\frac{\partial \lambda_{l}}{\partial \rho_{s}}=-\frac{\lambda_{l}}{\rho} \\
\frac{\partial \lambda_{l}}{\partial \mu}=\frac{1}{\mu} \lambda_{l} \\
\frac{\partial^{2} \lambda_{l}}{\partial k \partial \mu_{s}}=-\frac{2 k}{\rho} \\
\frac{\partial^{2} \lambda_{l}}{\partial k \partial \rho_{s}}=\frac{2 k \mu}{\rho^{2}} .
\end{gathered}
$$

These formulas are in agreement with taking direct derivatives of (31) with respect to the various parameters.

In the general case of an arbitrary number of layers over a half-space, the terms with $m \neq l$ do not cancel out. The success of this perturbation method relies on the rate of convergence of the various infinite series in equations (25) and (26) as the method cannot be economical if many terms have to be calculated. In order to speed up the convergence of these series, we introduced an infinitely rigid half-space beyond a certain depth where the displacements of the fundamental mode (or in general the $l$ th mode) are negligible, but where the displacements of higher modes are still noticeable. Thus the eigenvalues of the fundamental mode will not be different in the case of $N-1$ layers over a half-space, but those of the higher modes will be affected. This construction eliminates the continuous spectrum of (1) and (2) completely as the boundary value problem becomes a problem over a finite interval which has only discrete eigenvalues. For a large I the problem in essence becomes one of a single layer over a rigid half-space and from (31) one observes that in this case the eigenvalues grow like $l^{2}$. Hence for the large $l$ the series in (25), (26) behave like $\sum^{\infty} 1 / l^{2}$ and converge rapidly. Experience has shown that usually four or five modes give sufficient accuracy for the partials and often less modes are needed.

To test the method we chose two structures from Alexander (1963) representing two possible crustal models for the Basin and Range province (Figure 1a, b). The groupvelocity dispersion was calculated for these two models and then we checked whether we could predict one dispersion curve from the other, using the partial derivatives of the 


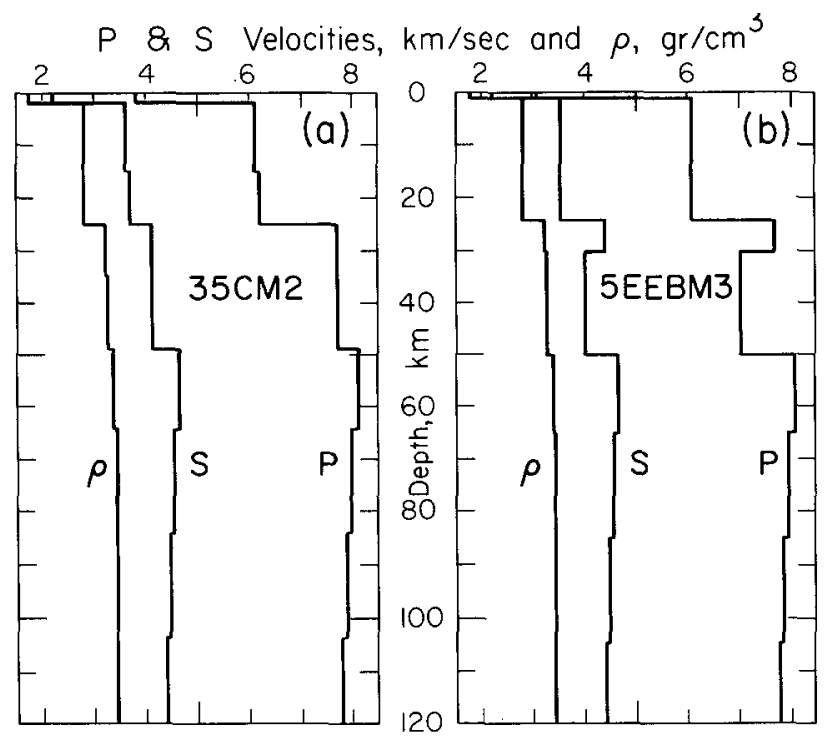

FIG. 1. (a) Velocity and density structure for the crustal model 35CM2 (from Alexander, 1963). (b) Velocity and density structure for the crustal model 5EEBM3 (from Alexander, 1963).

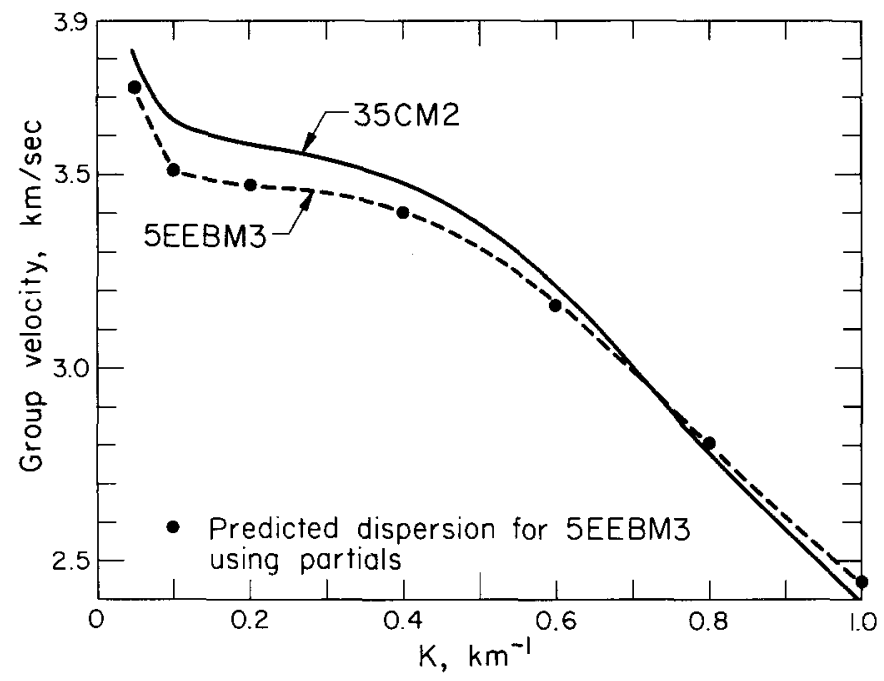

FIG. 2. Group-velocity dispersion for the $35 \mathrm{CM} 2$ and 5EEBM 3 crustal models.

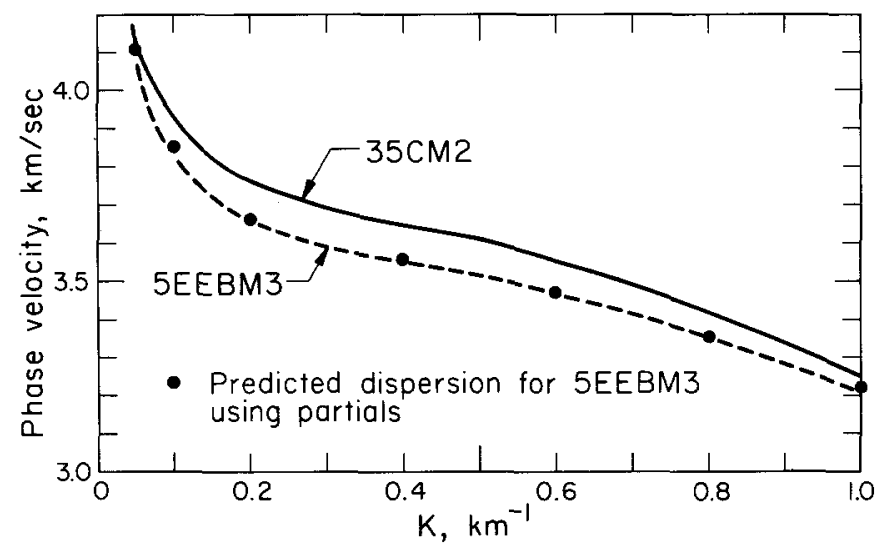

FIG. 3. Phase-velocity dispersion for the $35 \mathrm{CM} 2$ and 5EEBM 3 crustal models. 
group velocity (Figure 2). For comparison we included similar calculations for phase velocity (Figure 3). As the figures show, the group-velocity dispersion calculated by the partials matches the dispersion calculated directly quite well, and the partials do predict the crossing point of the two curves. This crossing is a result of the fact that the partials often change sign beyond certain depths. In the cases under discussion, this reversal occurred for high frequencies in some of the perturbed layers.

\section{ACKNOWLEDGMENTS}

The author wishes to thank Dr. D. Harkrider for his encouragement during this research. This research was supported by the Advanced Research Projects Agency of the Department of Defense and was monitored by the Air Force Office of Scientific Research under Contract F44620-72-C-0078.

\section{REFERENCES}

Alexander, S. (1963). Surface-wave propagation in the western United States, Ph.D. thesis, California Institute of Technology.

Anderson, D. L. (1964). Universal dispersion tables I, Love waves across oceans and continents on a spherical earth, Bull Seism. Soc. Am 54, 681-726.

Anderson, D. L. and C. B. Archambeau (1964). The anelasticity of the earth, J. Geophys. Res. 69, 2071-2084.

Anderson, D. L. and D. G. Harkrider (1968). Universal dispersion tables II, Variational parameters for amplitudes, phase velocity and group velocity for first four Love-wave modes for an oceanic and continental earth model, Bull. Seism. Soc. Am. 58, 1407-1500.

Coddington, E. A. and L. Levinson (1955). Theory of Ordinary Differential Equations, Chapters 7, 9, McGraw-Hill, New York.

Harkrider, D. G. (1968). The perturbation of Love-wave spectra, Bull. Seism. Soc. Am. 58, 861-880.

Landau, L. D. and E. M. Lifshitz (1965). Quantum Mechanics, Chapter VI, Pergamon Press, New York.

Takeuchi, H., J. Dorman, and M. Saito (1964). Partial derivatives of surface-wave phase velocity with respect to physical parameter changes within the earth, J. Geophys. Res. 69, 3429-3441.

Seismological Laboratory

California Institute of TeChnology

Pasadena, California, 91125

Contribution No. 2577, Division of Geological

and Planetary Sciences.

Manuscript received February 10, 1975 